\title{
ORIGINAL RESEARCH \\ MR Imaging of the Optic Nerve Sheath in Patients with Craniospinal Hypotension
}

\author{
A. Rohr \\ BACKGROUND AND PURPOSE: Craniospinal hyper- or hypotension leads to morphologic changes in \\ U. Jensen \\ certain intracranial structures. We tested the hypothesis that the amount of CSF in the ONS visible in \\ C. Riedel \\ MR imaging is reduced in patients with $\mathrm{CSH}$.
}

A. van Baalen

M.-C. Fruehauf

T. Bartsch

J. Hedderich

L. Doerner

O. Jansen

MATERIALS AND METHODS: Nineteen patients with CSH were prospectively studied. Three readers assessed the width of the peri-optical CSF rim at 4 different anatomic positions by using coronal STIR sequences from a 3T MR imaging scanner. The height of the pituitary gland was also measured. Results were compared with normal values obtained with the same imaging technique. Qualitative signs of $\mathrm{CSH}$ also recorded were engorgement of venous sinuses, dural enhancement, subdural effusion, narrow ventricles, and sagging brain.

RESULTS: CSF signal intensity surrounding the optic nerves was diminished in at least 2 of the 4 positions used for measurements so that decreased diameters of the ONSs were observed in all patients (sensitivity, 100\%; specificity, 97\%). The height of the pituitary gland was above normal limits in 12 of 19 patients (sensitivity, 63\%; specificity, 97\%). Frequencies of qualitative signs of CSH varied from $32 \%$ to $81 \%$.

CONCLUSIONS: The ISSON in patients with CSH is partially or fully collapsed due to reduced CSF content. In comparison with other anatomic markers, this sign showed the highest sensitivity for the diagnosis of patients with $\mathrm{CSH}$ in this study.

\begin{abstract}
ABBREVIATIONS: $\mathrm{Cl}=$ confidence interval; $\mathrm{CSH}=$ craniospinal hypotension; FLAIR = fluidattenuated inversion recovery; GRE = gradient-recalled echo; ICC = intraclass correlation coefficient; ISSON = intersheath space of the optic nerve; $L P=$ lumbar puncture; $M M=$ millimeter; $M R I=M R$ imaging; $M R V=3 D$ phase-contrast MR venography; $N=$ values within the normal range; N.A. = not applicable because of motion artifacts; N.M. = not measured; N.P. = contrastenhanced sequences were not performed; ONS = optic nerve sheath; STIR = short tau inversion recovery; $\mathrm{T} 1 \mathrm{WI}=\mathrm{T} 1$-weighted images; $\mathrm{T} 2 \mathrm{WI}=\mathrm{T} 2$-weighted images; $\mathrm{TSE}=$ turbo spin-echo; $\mathrm{VP}$ shunt $=$ ventriculoperitoneal shunt
\end{abstract}

C $\mathrm{SH}$ (also intracranial hypotension) is the result of CSF volume depletion, either due to dural rupture (spontaneous, traumatic, or iatrogenic) or CSF overdrainage in the case of VP shunt surgery. ${ }^{1-5}$ Patients typically complain of orthostatic headaches accompanied by various symptoms, such as neck stiffness, tinnitus, hearing impairment, photophobia, or nausea. ${ }^{6}$ In a substantial minority of patients, however, headaches are not orthostatic and may mimic other types of headaches. Some patients with CSH do not have headaches but display various neurologic symptoms. CSF opening pressure during LP is typically decreased in patients with CSH but can also be within the normal range. ${ }^{4}$ An LP itself_-despite being regarded as the diagnostic criterion standard-can cause $\mathrm{CSH}$ and is, therefore, not an ideal diagnostic procedure and should be avoided in these patients.

Neuroimaging supports the diagnosis of CSH, either by directly demonstrating a causative spinal CSF leak or by showing indirect signs of decreased intracranial or intraspinal pres-

Received July 29, 2009; accepted after revision March 17, 2010

From the Departments of Neuroradiology (A.R., U.J., C.R., M.-C.F., O.J.), Neuropediatrics (A.v.B.), Neurology (T.B.), Medical Computer Science and Statistics (J.H.), and Neurosurgery (L.D.), University Clinic Schleswig-Holstein Campus Kiel, Kiel, Germany.

A.R. and U.J. share first authorship.

Please address correspondence to Axel C. Rohr, MD, Department of Neuroradiology, University Clinic Schleswig-Holstein Campus Kiel, Arnold-Heller-Str 9, 24105 Kiel, Germany; e-mail: Axel.Rohr@gmx.de

DOI 10.3174/ajnr.A2120 sure. Several MR imaging signs associated with CSH have been described, including engorged venous sinuses, narrow ventricles, enhanced meninges, enlarged pituitary gland, subdural effusions, and sagging of the brain. ${ }^{1,7-12}$ A drawback of these signs is their lack of sensitivity, specificity, and/or objectivity. For example, the presence of engorged venous sinuses seems to be a sensitive sign indicative of $\mathrm{CSH},{ }^{8,10}$ but its evaluation is highly subjective because of normal interindividual variations. Similar problems are encountered when assessing the shape of the ventricles. ${ }^{3}$

Meningeal enhancement is also a nonspecific finding in patients with headaches caused by meningitis ${ }^{1,2}$ and can be lacking in patients with $\mathrm{CSH} .{ }^{11}$ Furthermore, contrast agent is not always used in the diagnostic imaging of patients with headache, which limits the usefulness of this sign. An enlarged pituitary gland has been associated with $\mathrm{CSH}^{7}$ but age- and sex-dependent variations in the size of the pituitary gland must be taken into consideration. ${ }^{13}$ Furthermore, the pituitary gland can also be enlarged in cases of hyperplasia or adenoma. Subdural effusion and sagging of the brain can be caused by $\mathrm{CSH}^{5,9}$ but the opposite is also frequently found: Subdural effusion can cause intracranial hypertension with subsequent sagging of the brain.

Due to the lack of specific clinical symptoms and neuroimaging signs, patients with $\mathrm{CSH}$ often are misdiagnosed and erroneously treated for other causes. Thus, CSH was only diagnosed correctly after multiple clinical examinations in $94 \%$ 


\begin{tabular}{|c|c|c|c|c|}
\hline $\begin{array}{l}\text { Patient } \\
\text { No. }\end{array}$ & Sex & $\begin{array}{l}\text { Age } \\
\text { (yr) }\end{array}$ & $\begin{array}{l}\text { Etiology } \\
\text { of CSH }\end{array}$ & $\begin{array}{c}\text { Opening } \\
\text { Pressure } \\
\text { on LP }\end{array}$ \\
\hline 1 & $\mathrm{M}$ & 72 & Idiopathic & Dry tap \\
\hline 2 & $M$ & 23 & Idiopathic & $11 \mathrm{~cm} \mathrm{H}_{2} \mathrm{C}$ \\
\hline 3 & M & 63 & Idiopathic & Dry tap \\
\hline 4 & $F$ & 40 & Idiopathic & Dry tap \\
\hline 5 & $\mathrm{~F}$ & 23 & Idiopathic & N.M. \\
\hline 6 & $F$ & 49 & Idiopathic & Dry tap \\
\hline 7 & $\mathrm{~F}$ & 59 & Idiopathic & Dry tap \\
\hline 8 & M & 39 & Post-LP & N.M. \\
\hline 9 & $F$ & 33 & Post-LP & N.M.. \\
\hline 10 & $\mathrm{~F}$ & 24 & Post-LP & N.M. \\
\hline 11 & $M$ & 70 & Post-LP & N.M. \\
\hline 12 & M & 29 & Post-LP & N.M. \\
\hline 13 & M & 18 & VP shunt & N.M. \\
\hline 14 & $\mathrm{~F}$ & 20 & VP shunt & N.M. \\
\hline 15 & $\mathrm{~F}$ & 18 & VP shunt & N.M. \\
\hline 16 & $F$ & 67 & VP shunt & N.M. \\
\hline 17 & $\mathrm{~F}$ & 44 & VP shunt & N.M. \\
\hline 18 & $\mathrm{~F}$ & 43 & VP shunt & N.M. \\
\hline 19 & $\mathrm{~F}$ & 35 & VP shunt & N.M. \\
\hline
\end{tabular}

a Patients were sorted according to the etiology of CSH.

of the patients in a former study. ${ }^{14}$ The correct diagnosis of $\mathrm{CSH}$-ideally accomplished by noninvasive methods — would not only help to avoid unnecessary risks but would also lead to effective treatment, such as a spinal peridural blood patch. ${ }^{6}$

The above-mentioned problems emphasize the need for a simple and reliable parameter for the diagnosis of CSH. Previous studies have shown that the diameter of the ONS might be a sensitive parameter for detection of patients with intracranial hypertension. ${ }^{15}$ In this condition, elevated intracranial CSF pressure leads to CSF inflow into the ISSON and subsequently to enlargement of the diameter of the ONS (ONS hydrops). In our experience, the opposite can be demonstrated in patients with CSH. These patients display abnormally little or even no CSF rim in the ONS, and this could be used as a sign of CSH. The aim of this study was to assess the reliability of this sign in patients with CSH by comparing their measurements of the ONS with the measurements in a healthy control population. ${ }^{13}$

\section{Materials and Methods}

\section{Patients}

We prospectively included 19 patients admitted consecutively to our departments of neurology and neurosurgery ( 7 men, 12 women; age range, 18-72 years; median age, 40 years; Table 1) who met the clinical diagnostic criteria of $\mathrm{CSH}$ according to the Headache Classification Subcommittee of the International Headache Society (Table 2). ${ }^{6}$ Five patients developed headache within 5 days after LP. In 7 patients, symptoms occurred spontaneously. In another 7 patients, symptoms developed after insertion of a VP shunt. CSF opening pressure during LP was measured in 6 patients in whom CSF analysis or myelography was indicated. It was low-to-normal in 1 patient $\left(11 \mathrm{~cm} \mathrm{H}_{2} \mathrm{O}\right.$, patient 2) and decreased in 5 patients. Exclusion criteria were the usual contraindications for MR imaging.

In all patients, cranial MR imaging was performed for clinical reasons. Nevertheless, written informed consent was obtained, including consent to add MR imaging sequences. Institutional review board approval was also obtained. All patients underwent a standardized cranial MR imaging between February 2006 and February 2009,
Table 2: Diagnostic criteria of CSH $^{\mathrm{a}}$

\section{All Patients}

A) Headache that worsens within 15 minutes after sitting or standing and improves after lying, with at least 1 of the following criteria:

1) Neck stiffness, 2) tinnitus, 3) hypacusis, 4) photophobia, 5) nausea Subgroup of patients with post-LP syndrome

B) Headache develops within 5 days after dural puncture

C) Headache resolves either

1) Spontaneously within 1 week

2) Within 48 hours after effective treatment of the spinal fluid leak Subgroup of patients with idiopathic low CSF pressure syndrome

B) At least 1 of the following:

1) CSF opening pressure $<60 \mathrm{~mm} \mathrm{H}_{2} \mathrm{O}$ in sitting position

2) Evidence of CSF leakage on conventional myelography, CT myelography, or cisternography

3) Evidence of low CSF pressure on MRI (eg, pachymeningeal enhancement)

C) No history of dural puncture or other cause of CSF fistula

D) Headache resolves after epidural blood patching

Subgroup of patients with shunt overdrainage

B) Previous shunt operation

C) Evidence of low CSF pressure on MRI (eg, pachymeningeal enhancement)

${ }^{a}$ Adapted with permission from the Headache Classification Subcommittee of the International Headache Society. ${ }^{6}$

according to the protocol mentioned below. None of the patients had contraindications (claustrophobia, cardiac pacemakers, or other metallic implants). In all 5 patients with post-LP syndrome, headache resolved with conservative treatment (bedrest, intravenous infusion of half-isotonic sodium chloride solution, and theophylline administration). The same treatment was effective in 3 of the 7 patients with spontaneous CSH. In the remaining 4 patients with spontaneous $\mathrm{CSH}$, peridural spinal blood patches were required. In none of the 7 patients with overdrainage of the VP shunt were headaches severe. Therefore, shunt revisions were not indicated, but headaches did resolve after adjustment of a shunt valve in 1 patient.

\section{Controls}

The results of measurements of the ONS in 123 individuals without intracranial hypo- or hypertension, by using identical soft- and hardware, MR imaging protocols, and neuroradiologic readers, were used for comparison. Results of our control group have previously been published. ${ }^{13}$ Because no correlations of the ONS diameters with sex or age were found in this study, patients and controls were not matched for these characteristics.

\section{MR Imaging}

Cranial MR imaging was performed in all individuals on a 3T scanner (Achieva, Philips Healthcare, Best, the Netherlands; SENSE Head coil, 8 elements, Philips Healthcare) by using a standardized protocol that included at least the following sequences: T2WI TSE, axial (TR/TE, 4456/100 ms; section thickness, $3 \mathrm{~mm}$ ), MRV (TR/TE, $18 / 6.5 \mathrm{~ms}$; section thickness, $0.8 \mathrm{~mm}$; velocity encoding, $12 \mathrm{~cm} / \mathrm{s}$ ), STIR (TR/TE/TI, 3709/80/200 ms; section thickness, $3 \mathrm{~mm}$ ) covering the orbit and the pituitary gland in the coronal plane, sagittal FLAIR (TR/TE/TI, 12000/160/2850 ms; section thickness, $3 \mathrm{~mm}$ ), and T1WI axial GRE (TR/TE, 319/22.3 ms; section thickness, 4 mm). In 12 patients, contrast-enhanced T1WI GRE sequences were also performed.

\section{MR Image Analysis}

The vertical diameter of the ONS - including the CSF rim in the ISSON—and the vertical diameter of the optic nerve without the CSF 


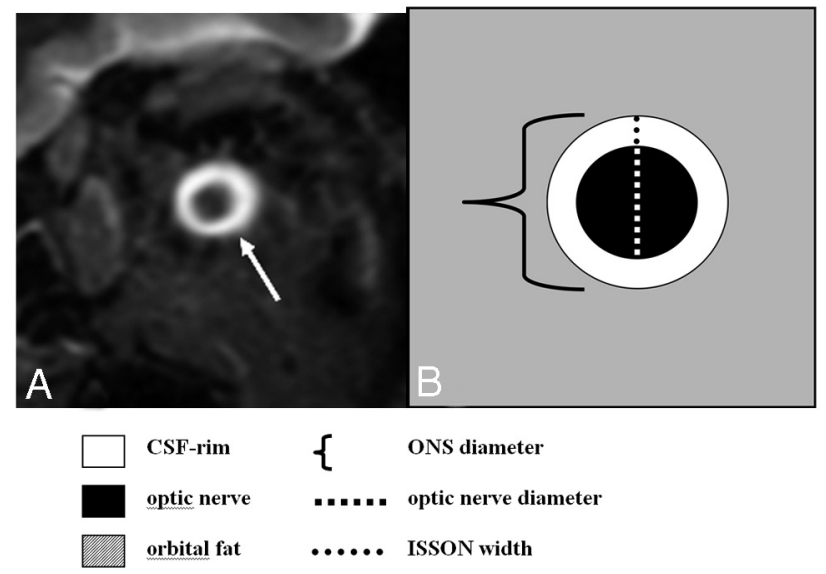

Fig 1. Measurement of the ONS. A, Coronal STIR sequence of the orbital contents shows the normal appearance of the optic nerve surrounded by a high-signal-intensity rim representing CSF in the ISSON (arrow). B, Schematic drawing of the ONS including the optic nerve and surrounding CSF rim.

rim were measured at 4 intraorbital positions $(3,6,10$, and $20 \mathrm{~mm}$ behind the eyeball; Fig 1). The width of the ISSON was assessed by subtraction of both measurements and subsequent division by 2 . The intraorbital positions were specified by cross-referencing on axial T2WIs. Because there was no difference in the diameters between the left and right ONS, only the left ONS of patients was chosen for analysis (1 measurement at each position). A pathologic diameter was defined as any value below the third percentile of the normal range ( $1.0 \mathrm{~mm}$ in position 1 , and $0.9 \mathrm{~mm}$ in positions $2-4$ ). If a CSF rim around the optic nerve was not visible, the ISSON was defined as "collapsed." Irrespective of the quantitative measurements, readers were asked to visually judge whether the ISSON width in patients was pathologically narrow or collapsed along its entire course during a second reading (visual inspection). In all patients, the height of the pituitary gland was measured on coronal STIR images and was compared with previously published sex- and age-adjusted normal values (maximum normal pituitary gland height: $5.6 \mathrm{~mm}$ [men], $6.9 \mathrm{~mm}$ [women 16-40 years of age], $5.8 \mathrm{~mm}$ [women 41-62 years of age], and $6.0 \mathrm{~mm}$ [women $>62$ years of age]). ${ }^{13}$

We also subjectively assessed the presence of venous sinus engorgement in maximum intensity projections of MRV, the presence of narrow ventricles in T2WIs, the presence of meningeal enhancement in patients who received contrast-enhanced T1WIs, the presence of subdural effusions, and the presence of brain sagging in FLAIR images of our patient population. All measurements were analyzed by 3 readers: 1) A.R., experienced board-certified radiologist and neuroradiologist; 2) U.J., Specialist Registrar in neuroradiology, 3) M.-C.F., senior medical student who was trained to perform measurements of the ONS on MR imaging. The results of the first reader were used for the primary analysis. The results of all 3 readers were used to calculate interobserver reliabilities.

\section{Statistics}

Images were randomly assigned to the 3 readers, and data were stored on Excel spreadsheets (Microsoft, Bothell, Washington). Statistical analysis was done by using the software tools Statistical Package for the Social Sciences, Version 15 (SPSS, Chicago, Illinois) and R package, Release 2.11.0 (open source software, The R Foundation for Statistical Computing, Vienna, Austria). The Mann-Whitney $U$ test was used to prove differences between values of measurements in patients with $\mathrm{CSH}$ and controls. The diagnostic sensitivity (including 95\% CIs) was assessed to compare the results of ONS measurements and different MR imaging signs of CSH. In individuals with collapsed ISSONs, the width of the ISSON was defined as zero in the statistical analysis. To determine the degree of interobserver reliability in visual interpretation of nonquantitative features of CSH (engorgement of venous sinuses, narrow ventricles, dural enhancement, subdural effusion, brain sagging, and narrow or collapsed ISSON), we calculated $\kappa$ values. Likewise, the ICC was computed to determine the degree of the interobserver reliability for the quantitative values (width of the ISSON and height of the pituitary gland).

\section{Results}

The ISSON widths of patients differed significantly from those of controls as a result of diminished CSF. Values of measurements are listed in Table 3. Collapsed ISSON was observed with a sensitivity of $44 \%$ in position 1 ( $3 \mathrm{~mm}$ behind the eyeball), $74 \%$ in position 2 ( $6 \mathrm{~mm}$ behind the eyeball), $74 \%$ in position $3(10 \mathrm{~mm}$ behind the eyeball), and $84 \%$ in position 4 (20 mm behind the eyeball); it was not observed in any patient from the control population (specificity, 100\%). A reduction in the ISSON width below normal limits or a collapsed ISSON was detected with sensitivities of $61 \%, 84 \%, 100 \%$, and $100 \%$ in positions $1-4$, respectively. Specificity was $97 \%$ for each position. In $8 / 19$ patients (42\%) with CSH, the ISSON was collapsed along its entire length. In the remaining 11 patients (58\%), we found only residual CSF signal intensity predominantly in the most frontal part of the ISSON. On visual interpretation, all 3 readers agreed that the width of the ISSON was abnormally narrow or that the ISSON was entirely collapsed in all patients.

The height of the pituitary gland was significantly larger in symptomatic patients $(6.9 \pm 2.3 \mathrm{~mm})$ than in the control population $(4.3 \pm 0.9 \mathrm{~mm}, P=.001)$. It was above the normal limit in 12 of 19 patients (sensitivity, 63\%; specificity, 97\%; Table 4). An example of a "collapsed ONS" and enlargement of the pituitary gland in a patient with $\mathrm{CSH}$ and follow-up imaging after treatment is shown in Fig 2.

Descriptive imaging features of CSH were recorded but were not matched with a control group. Frequencies for these features were $84 \%$ for venous sinus engorgement, $79 \%$ for dural enhancement, $68 \%$ for subdural effusion, $47 \%$ for narrow ventricles, and $32 \%$ for brain sagging (Table 4 ). At least 1 of these features was present in 17 of 19 patients (89\%).

The interobserver reliability was high regarding measurements of the pituitary gland height $($ ICC $=0.974)$. It was also high regarding measurements of the ISSON width, with the ICC being better in the anterior aspect (ICC $=0.908$ in position 1 ) than in the posterior aspect of the ONS (ICC $=0.824$ in position 4). The interobserver reliabilities regarding nonquantitative features of CSH were best for the features "presence of subdural hygromas" and "presence of abnormally narrow ISSON" ( $\kappa=1.0$ each) and worst for the feature "presence of narrow ventricles" ( $\kappa=0.579$, Tables 3 and 4 ).

The number of patients was too small to allow a meaningful statistical analysis of patient subgroups. There seems to be a tendency for our patients with post-LP syndrome to exhibit some features less often than patients with idiopathic CSH or patients with overdrainage of a VP shunt (narrow ventricles [20\% versus 
Table 3: Results of the analysis of ISSON width in 19 patients with CSH $^{\mathrm{a}, \mathrm{b}}$

ISSON Width

\begin{tabular}{|c|c|c|c|c|c|c|c|c|c|}
\hline \multirow{3}{*}{$\begin{array}{l}\text { Patient } \\
\text { No. }\end{array}$} & \multicolumn{8}{|c|}{ ISSON Width } & \multirow{3}{*}{$\begin{array}{c}\text { ISSON Width } \\
\frac{\text { (Gross Visual Inspection) }}{-/ \mathrm{N}}\end{array}$} \\
\hline & \multicolumn{2}{|c|}{ Position 1} & \multicolumn{2}{|c|}{ Position 2} & \multicolumn{2}{|c|}{ Position 3} & \multicolumn{2}{|c|}{ Position 4} & \\
\hline & $-/ N$ & MM & $-/ N$ & MM & $-/ \mathrm{N}$ & MM & $-/ \mathrm{N}$ & MM & \\
\hline 1 & $\mathrm{~N}$ & 1.1 & - & 0 & - & 0 & - & 0 & - \\
\hline 2 & - & N.A. & - & 0.6 & - & 0.6 & - & 0.7 & - \\
\hline 3 & $\mathrm{~N}$ & 1.2 & $\mathrm{~N}$ & 1.1 & - & 0.7 & - & 0.8 & - \\
\hline 4 & - & 0 & - & 0 & - & 0 & - & 0 & - \\
\hline 5 & - & 0 & - & 0 & - & 0 & - & 0 & - \\
\hline 6 & - & 0 & - & 0 & - & 0 & - & 0 & - \\
\hline 7 & - & 0 & - & 0 & - & 0 & - & 0 & - \\
\hline 8 & - & 0 & - & 0 & - & 0 & - & 0 & - \\
\hline 9 & $\mathrm{~N}$ & 1.6 & $\mathrm{~N}$ & 0.9 & - & 0.7 & - & 0 & - \\
\hline 10 & $\mathrm{~N}$ & 1.2 & - & 0.6 & - & 0.7 & - & 0 & - \\
\hline 11 & - & 0.8 & - & 0 & - & 0 & - & 0.8 & - \\
\hline 12 & $\mathrm{~N}$ & 1.1 & - & 0 & - & 0 & - & 0 & - \\
\hline 13 & - & 0 & - & 0 & - & 0 & - & 0 & - \\
\hline 14 & $\mathrm{~N}$ & 1.2 & - & 0 & - & 0 & - & 0 & - \\
\hline 15 & - & 0.8 & - & 0 & - & 0 & - & 0 & - \\
\hline 16 & - & 0.7 & - & 0 & - & 0 & - & 0 & - \\
\hline 17 & - & 0 & - & 0 & - & 0 & - & 0 & - \\
\hline 18 & $\mathrm{~N}$ & 1.4 & $\mathrm{~N}$ & 1.2 & - & 0.7 & - & 0 & - \\
\hline 19 & - & 0 & - & 0 & - & 0 & - & 0 & - \\
\hline Mean & \multicolumn{2}{|c|}{0.6} & \multicolumn{2}{|c|}{0.2} & \multicolumn{2}{|c|}{0.2} & \multicolumn{2}{|c|}{0.1} & \\
\hline SD & \multicolumn{2}{|c|}{0.6} & \multicolumn{2}{|c|}{0.4} & \multicolumn{2}{|c|}{0.3} & \multicolumn{2}{|c|}{0.3} & \\
\hline Sensitivity & \multicolumn{2}{|c|}{$61 \%$} & \multicolumn{2}{|c|}{$84 \%$} & \multicolumn{2}{|c|}{$100 \%$} & \multicolumn{2}{|c|}{$100 \%$} & $100 \%$ \\
\hline Cl $(95 \%)$ & \multicolumn{2}{|c|}{$36 \%-83 \%$} & \multicolumn{2}{|c|}{$60 \%-97 \%$} & \multicolumn{2}{|c|}{$85 \%-100 \%$} & \multicolumn{2}{|c|}{$85 \%-100 \%$} & $85 \%-100 \%$ \\
\hline $\mathrm{ICC}^{\mathrm{C}}$ & \multicolumn{2}{|c|}{0.908} & \multicolumn{2}{|c|}{0.859} & \multicolumn{2}{|c|}{0.862} & \multicolumn{2}{|c|}{0.824} & \\
\hline$\kappa^{\mathrm{c}}$ & & & & & & & & & 1 \\
\hline
\end{tabular}

a Measurements were obtained in 4 intraorbital positions approximately 3, 6, 10, and $20 \mathrm{~mm}$ behind the eyeball. Specificity was $97 \%$ each.

b -indicates decreased ( $<1.0 \mathrm{~mm}$, [position 1]; $<0.9 \mathrm{~mm}$, [positions 2-4]). Regarding gross visual inspection,-corresponds to a reduction or absence of CSF in the ISSON

${ }^{\mathrm{c}} \mathrm{ICC}$ and $\kappa$ values were computed for results of 3 readers.

\begin{tabular}{|c|c|c|c|c|c|c|c|}
\hline \multirow{2}{*}{$\begin{array}{l}\text { Patient } \\
\text { No. }\end{array}$} & \multicolumn{2}{|c|}{$\begin{array}{c}\text { Increased } \\
\text { Pituitary Height }\end{array}$} & \multirow{2}{*}{$\begin{array}{c}\begin{array}{c}\text { Engorged } \\
\text { Venous Sinuses }\end{array} \\
+/ \mathrm{N}\end{array}$} & \multirow{2}{*}{$\begin{array}{c}\text { Narrow } \\
\text { Ventricles } \\
+/ N\end{array}$} & \multirow{2}{*}{$\begin{array}{c}\begin{array}{c}\text { Dural } \\
\text { Enhancement }\end{array} \\
+/ \mathrm{N}\end{array}$} & \multirow{2}{*}{$\begin{array}{c}\begin{array}{c}\text { Subdural } \\
\text { Effusion }\end{array} \\
+/ \mathrm{N}\end{array}$} & \multirow{2}{*}{$\begin{array}{c}\text { Brain } \\
\text { Sagging } \\
+\end{array}$} \\
\hline & $+/ \mathrm{N}$ & $\mathrm{MM}$ & & & & & \\
\hline 1 & $\mathrm{~N}$ & 5.2 & $\mathrm{~N}$ & + & + & + & $\mathrm{N}$ \\
\hline 2 & $\mathrm{~N}$ & 3.8 & $\mathrm{~N}$ & $\mathrm{~N}$ & $\mathrm{~N}$ & $\mathrm{~N}$ & $\mathrm{~N}$ \\
\hline 3 & $\mathrm{~N}$ & 3.8 & + & $\mathrm{N}$ & + & + & + \\
\hline 4 & $\mathrm{~N}$ & 6.3 & + & + & + & + & + \\
\hline 5 & + & 11.7 & + & $\mathrm{N}$ & + & + & + \\
\hline 6 & + & 8.0 & + & + & N.P. & + & + \\
\hline 7 & + & 6.7 & + & + & N.P. & + & $\mathrm{N}$ \\
\hline 8 & + & 6.0 & + & $\mathrm{N}$ & N.P. & $\mathrm{N}$ & $\mathrm{N}$ \\
\hline 9 & + & 8.3 & + & + & + & + & $\mathrm{N}$ \\
\hline 10 & + & 8.1 & + & $\mathrm{N}$ & $\mathrm{N}$ & $\mathrm{N}$ & $\mathrm{N}$ \\
\hline 11 & $\mathrm{~N}$ & 3.9 & $\mathrm{~N}$ & $\mathrm{~N}$ & $\mathrm{~N}$ & $\mathrm{~N}$ & $\mathrm{~N}$ \\
\hline 12 & + & 7.7 & + & $\mathrm{N}$ & + & + & $\mathrm{N}$ \\
\hline 13 & $\mathrm{~N}$ & 3.0 & + & $\mathrm{N}$ & + & + & $\mathrm{N}$ \\
\hline 14 & $\mathrm{~N}$ & 6.4 & + & + & N.P. & + & $\mathrm{N}$ \\
\hline 15 & + & 7.1 & + & + & N.P. & $\mathrm{N}$ & $\mathrm{N}$ \\
\hline 16 & + & 9.6 & + & $\mathrm{N}$ & + & $\mathrm{N}$ & + \\
\hline 17 & + & 7.6 & + & + & + & + & $\mathrm{N}$ \\
\hline 18 & + & 9.6 & + & + & + & + & $\mathrm{N}$ \\
\hline 19 & + & 7.8 & + & $\mathrm{N}$ & + & + & + \\
\hline Frequency & \multicolumn{2}{|c|}{$63 \%$} & $84 \%$ & $47 \%$ & $79 \%$ & $68 \%$ & $32 \%$ \\
\hline $95 \% \mathrm{Cl}$ & \multicolumn{2}{|c|}{$38 \%-84 \%$} & $60 \%-97 \%$ & $24 \%-71 \%$ & $49 \%-95 \%$ & $43 \%-87 \%$ & $13 \%-57 \%$ \\
\hline$\kappa^{\mathrm{C}}$ & & & 0.775 & 0.579 & 0.869 & 1 & 0.652 \\
\hline $\mathrm{ICC}^{\mathrm{C}}$ & \multicolumn{2}{|c|}{0.974} & & & & & \\
\hline
\end{tabular}

57\%], dural enhancement [50\% versus 90\%], subdural effusion [29\% versus $71 \%$, and brain sagging [0\% versus $43 \%]$ ).
Follow-up MR images were available in 6 patients. Two of those patients with idiopathic $\mathrm{CSH}$ received epidural blood 

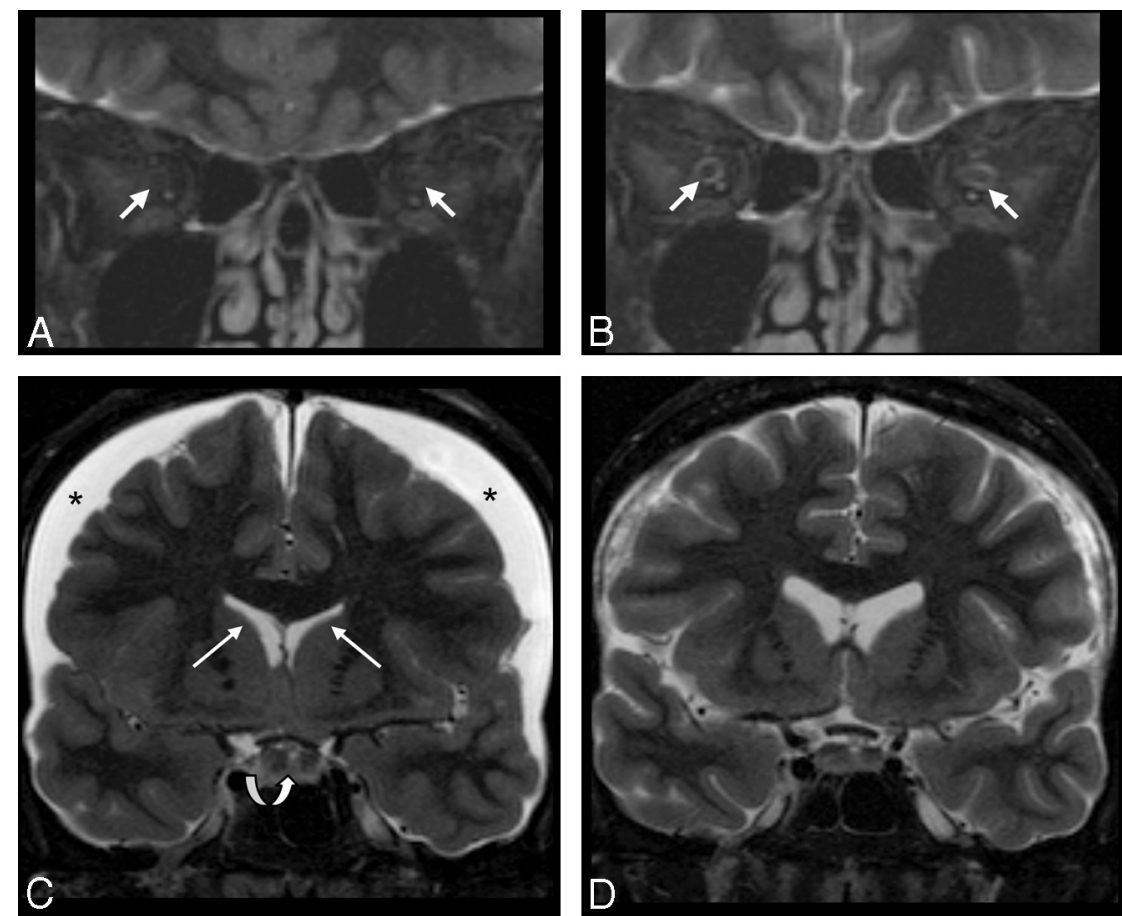

Fig 2. Patient sample 1. Cranial MR imaging (coronal STIR sequence) of a 49-year-old woman (patient 6) with CSH due to spontaneous CSF leak in the spinal dura before (A and C) and on follow-up imaging after $(B$ and $D)$ successful therapy with an epidural blood patch. Imaging of the orbital contents reveals a lack of the normal peri-optical CSF rim in the ISSON (arrows in $A$, collapsed ONS), which normalized with therapy (arrows in B). Width of the ISSON is $1.3 \mathrm{~mm}$ (position 1), $1.3 \mathrm{~mm}$ (position 2), $1.0 \mathrm{~mm}$ (position 3), and $0.9 \mathrm{~mm}$ (position 4) on follow-up. Lower normal width of the ISSON is $1 \mathrm{~mm}$ (position 1) and $0.9 \mathrm{~mm}$ (positions 2-4). C. Subdural effusion (asterisks), narrow ventricles (white arrows), and an enlarged pituitary gland (curved arrow) are in agreement with CSH. Following therapy, the subdural effusion and the pituitary gland appear smaller and the ventricles regain normal size (D).
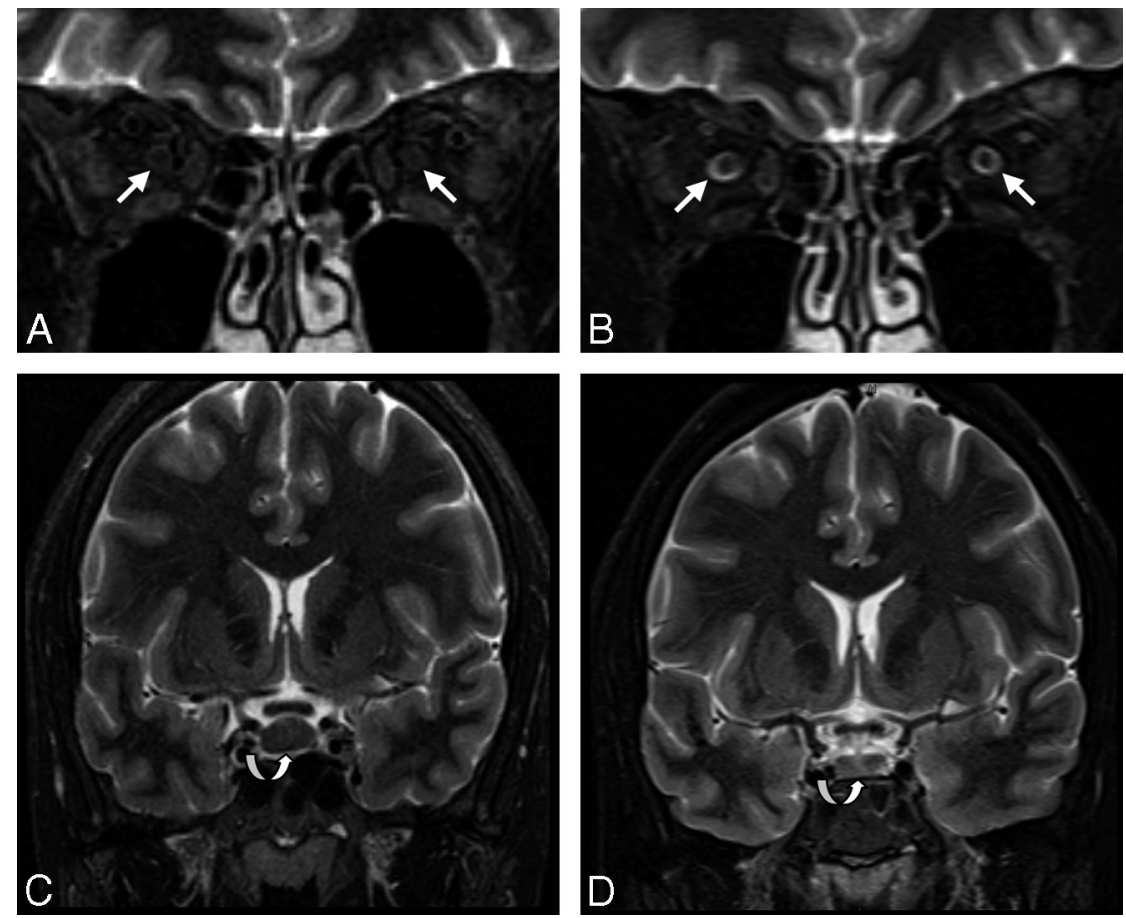

Fig 3. Patient sample 2. MR imaging of a 33-year-old woman (patient 9) with post-LP syndrome before ( $A$ and $C$ ) and on follow-up after successful conservative treatment (B and $D$ ). CSF in the ONSs is reduced to a minimum amount, normalizing after treatment (arrows in $A$ and $B$ ). The pituitary gland is enlarged (8.3 mm in height), regaining normal size on follow-up (curved arrows in $C$ and $D$ ). Very thin subdural hygromas could only be detected by FLAIR imaging (not shown). There is a minimal increase in ventricle size $(D)$ after treatment.

patches. Follow-up MR imaging showed normalization of the ONS in both patients (Fig 2). In another 2 patients who received epidural blood patches, headaches resolved but follow-up MR images were not available. Normalization of the ONS was also documented with MR imaging in 1 patient with
post-LP syndrome (Fig 3). In 1 patient with a VP shunt, ONS normalized on follow-up MR imaging after valve adjustment. The ONS of 2 patients with shunts whose valves could not be changed remained pathologic on follow-up. The clinical features of these patients did not change. 


\section{Discussion}

In the present study, we tested the hypothesis that the width of the ISSON is reduced in patients with CSH due to a lack of normal CSF accumulation. The normal width of the CSF rim in the ISSON as measured by cranial MR imaging in our control population ranges from $1.4 \pm 0.3 \mathrm{~mm}$ (anterior) to $1.0 \pm$ $0.2 \mathrm{~mm}$ (posterior). ${ }^{13}$ According to the Monro-Kellie hypothesis, CSF hypovolemia caused by spinal CSF leakage results in lower intracranial pressure so that the intracranial/extracranial pressure gradient is reduced or nonexistent. ${ }^{3}$ As a result, CSF outside the cranial space (ie, in the orbit) should be expected to be diminished in $\mathrm{CSH}$. We observed this phenomenon in all our patients with CSH. CSF in the ISSON was either reduced (predominantly in the anterior aspects of the ONS) or it was absent (predominantly in the posterior aspects of the ONS). Taking into account a lower normal cutoff value of $0.9 \mathrm{~mm}$ for the width of ISSON, we could predict CSH with a sensitivity of $100 \%$ and a specificity of $97 \%$ in our patient population when the middle and dorsal positions (positions 3 and 4) were evaluated.

A more subjective approach of visually judging a reduction in the amount of CSF or lack of CSF in the ISSON in patients with CSH has its practical merits as well: There was excellent agreement among our 3 readers, all experienced in orbital imaging, that the amount of CSF was pathologically reduced or absent in all patients of our cohort. Our findings are in accordance with a case series of 3 patients with CSH in whom decreased ONS diameters were reported. ${ }^{16}$ In that publication, however, a different MR imaging technique was used (fatsaturated T2WIs with a $1.5 \mathrm{~T}$ magnet) and the total ONS diameters were measured at only 1 position just behind the eyeball. Our study shows that measuring the CSF rim in the ISSON at that position can lead to false-negative results.

In our opinion, the sign of the collapsed ISSON has advantages in comparison with other reported MR imaging signs of CSH: It is sensitive and, because normal limits of the width of the ONS are known, less subjective. Enlargement of the pituitary gland in patients with $\mathrm{CSH}$ - the second sign we assessed quantitatively - was observed with a sensitivity of $63 \%$ and a specificity of $97 \%$ by using age- and sex-adjusted normal cutoff values. Because the main differential diagnosis, namely pituitary hyperplasia, is rare, we believe that this sign is also very useful in the work-up of patients with suspected $\mathrm{CSH}$. The etiology of this enlargement, however, is not fully understood. We assume that edema with consecutive enlargement of the pituitary gland could result from low or negative pressure in the sella turcica.

Recently, it has been reported that distended epidural veins and epidural fluid accumulation detected in spinal MR imaging might be more sensitive in the diagnosis of CSH than cranial MR imaging, with reported sensitivities of 95\% versus $83 \%$, respectively. ${ }^{17}$ Our results indicate that cranial MR im- aging can be even more sensitive than spinal MR imaging when high-contrast and high-resolution orbital imaging is applied. The STIR sequence perpendicular to the optic nerve used in our protocol (duration of 3 minutes 35 seconds) can be easily included in routine cranial MR imaging scans. Because it allows measurement of both the ONS diameter and the height of the pituitary gland, this sequence can be used to deliver 2 important parameters for the diagnosis of $\mathrm{CSH}$.

\section{Conclusions}

In patients with CSH, CSF content in the ISSONs is absent or reduced to below a width of $0.9 \mathrm{~mm}$ on coronal $3 \mathrm{~T}$ images (collapse of the ONS). This phenomenon lends itself well to visual interpretation on fat-suppressed sequences and can be regarded as a new sign of CSH. Our data suggest that the sensitivity and specificity of this sign might be higher than that of other known features. Including orbital imaging in the work-up of patients with CSH might, therefore, increase the accuracy of the diagnosis.

\section{References}

1. Chung SJ, Kim JS, Lee MC. Syndrome of cerebral spinal fluid hypovolemia: clinical and imaging features and outcome. Neurology 2000;55:1321-27

2. Grimaldi D, Mea E, Chiapparini L, et al. Spontaneous low cerebrospinal pressure: a mini review. Neurol Sci 2004;25(suppl 3):S135-37

3. Mokri B. Spontaneous intracranial hypotension. Curr Neurol Neurosci Rep 2001;1:109-17

4. Mokri B. Headaches caused by decreased intracranial pressure: diagnosis and management. Curr Opin Neurol 2003;16:319-26

5. Schievink WI. Spontaneous spinal cerebrospinal fluid leaks and intracranial hypotension. JAMA 2006;295:2286-96

6. Headache Classification Subcommittee of the International Headache Society. The International Classification of Headache Disorders: 2nd ed. Cephalalgia 2004;24(suppl 1):9-160

7. Alvarez-Linera J, Escribano J, Benito-Leon J, et al. Pituitary enlargement in patients with intracranial hypotension syndrome. Neurology 2000;55:1895-97

8. Farb RI, Forghani R, Lee SK, et al. The venous distension sign: a diagnostic sign of intracranial hypotension at MR imaging of the brain. AJNR Am JNeuroradiol 2007;28:1489-93

9. Hejazi N, Al-Witry M, Witzmann A. Bilateral subdural effusion and cerebra displacement associated with spontaneous intracranial hypotension: diagnostic and management strategies-report of two cases. J Neurosurg 2002; 96:956-59

10. Koss SA, Ulmer JL, Hacein-Bey L. Angiographic features of spontaneous intracranial hypotension. AJNR Am J Neuroradiol 2003;24:704-06

11. Schievink WI, Tourje J. Intracranial hypotension without meningeal enhancement on magnetic resonance imaging: case report. J Neurosurg 2000;92:475-77

12. Schoffer KL, Benstead TJ, Grant I. Spontaneous intracranial hypotension in the absence of magnetic resonance imaging abnormalities. Can J Neurol Sci 2002;29:253-57

13. Rohr A, Riedel C, Reimann G, et al. Pseudotumor cerebri: quantitative in-vivo measurements of markers of intracranial hypertension [in German]. Rofo 2008;180:884-90

14. Schievink WI. Misdiagnosis of spontaneous intracranial hypotension. Arch Neurol 2003;60:1713-18

15. Watanabe A, Kinouchi H, Horikoshi T, et al. Effect of intracranial pressure on the diameter of the optic nerve sheath. J Neurosurg 2008;109:255-58

16. Watanabe A, Horikoshi T, Uchida M, et al. Decreased diameter of the optic nerve sheath associated with CSF hypovolemia. AJNR Am J Neuroradiol 2008;29:863-64

17. Watanabe A, Horikoshi T, Uchida M, et al. Diagnostic value of spinal MR imaging in spontaneous intracranial hypotension syndrome. AJNR Am J Neuroradiol 2009;30:147-51 